\title{
Time-dependent localized Hartree-Fock density-functional linear response approach for photoionization of atomic excited states
}

\author{
Zhongyuan Zhou and Shih-I Chu \\ Department of Chemistry, University of Kansas, Lawrence, Kansas 66045, USA \\ (Received 7 February 2009; published 13 May 2009)
}

\begin{abstract}
We present a time-dependent localized Hartree-Fock density-functional linear response approach for the treatment of photoionization of atomic systems. This approach employs a spin-dependent localized HartreeFock exchange potential to calculate electron orbitals and kernel functions, and thus can be used to study the photoionization from atomic excited states. We have applied the approach to the calculation of photoionization cross sections of Ne ground state. The results are in agreement with available experimental data and have comparable accuracies with other $a b$ initio theoretical results. We have also extended the approach to explore the photoionization from $\mathrm{Ne}$ excited states and obtained some results for the photoionization from outer-shell and inner-shell excited states.
\end{abstract}

DOI: 10.1103/PhysRevA.79.053412

PACS number(s): 32.80.Fb, 31.15.ee, 31.15.es, 32.80.Zb

\section{INTRODUCTION}

Making use of local potentials and independent-particle response functions, density-functional theory (DFT) combined with linear response approximation (LRA) [1,2] has been successfully applied to study dynamic processes, such as photoabsorption [3-10] and dynamic polarizability [11-15], of atomic and molecular systems. The most attractive features of such an approach are its satisfactory accuracy and computational simplicity and efficiency. However, since the conventional DFT using traditional exchange-correlation (XC) potentials obtained from uniform electron gas, such as local-density approximation (LDA) [16,17] and generalized gradient approximation (GGA) [17-19], is a ground-state approach, the conventional DFT-LRA approach can only be used to investigate the dynamic processes associated with the ground state of a system. Even so, the counterpart of the DFT-LRA approach for the excited states has not yet been reported.

The difficulty encountered in the extension of the conventional DFT-LRA approach to the excited states stems from the XC potential used to characterize the excited states. A qualified $\mathrm{XC}$ potential for the excited states is required to be symmetry-dependent and self-interaction free and have a correct long-range behavior. The symmetry (such as electronic configuration, electron orbital angular momentum, and electron spin) of the XC potential is used to distinguish a state from the others, the self-interaction-free property of the $\mathrm{XC}$ potential is used to make the calculation of electron orbital energy accurate, and the correct asymptotic behavior of the XC potential is used to guarantee the Rydberg virtual orbitals which play a key role in autoionization resonances [7].

Recently, a localized Hartree-Fock (LHF) densityfunctional approach has been proposed and successfully applied to the ground-state calculation of atomic and molecular systems [20]. In this approach, the LHF exchange potential is self-interaction free and exhibits the correct long-range behavior. It only needs occupied orbitals and depends on the orbital symmetry of the state. More recently, a spindependent localized Hartree-Fock (SLHF) density-functional approach has been developed for the excited-state calculation of atomic and molecular systems [21]. This approach together with Slater's diagonal sum rule [22] have been successfully used to calculate the energies of multiply excited states of valence electrons of atomic systems [21] and the energies of inner-shell excited states of closed-shell [23] and open-shell [24] atomic systems.

In this paper, we present a time-dependent localized Hartree-Fock density-functional linear response approach for the treatment of photoionization from atomic excited states by combining the SLHF DFT with LRA. In this approach, the SLHF exchange potential is employed to calculate both the Kohn-Sham (KS) electron orbitals and kernel functions. This is different from the approach used in Ref. [10] where the LHF potential was only used to calculate the electron orbitals. We have applied this approach to the calculation of photoionization cross sections (PICS) of Ne ground state and extended it to the computation of PICS of Ne excited states.

\section{THEORETICAL METHODOLOGY}

\section{A. Linear response approximation of photoionization}

Suppose that an atomic system is in a time-dependent external field along $z$ axis $\mathcal{E}(t)=\mathcal{E}_{0} e^{-i \omega t}$, where $\mathcal{E}_{0}$ is the amplitude and $\omega$ the frequency. In dipole approximation, the interaction potential of an electron and the external field is $\phi^{\text {ext }}(\mathbf{r}, t)=\phi^{\text {ext }}(\mathbf{r}, \omega) e^{-i \omega t}$, where $\phi^{\text {ext }}(\mathbf{r}, \omega)=z \mathcal{E}_{0}$. Presence of the external field will induce a perturbation to the system and produce a redistribution of electron density [3]. In the case of weak field considered here, the dynamic response of the system has the same time dependence $e^{-i \omega t}$ as the field [8] and thus can be described by the LRA [25]. In frequency domain, total perturbing potential that an electron experiences can be expressed, in spin-dependent DFT framework [3,6-8], as

$$
\phi_{\sigma}^{S C F}(\mathbf{r}, \omega)=\phi^{e x t}(\mathbf{r}, \omega)+\sum_{\sigma^{\prime}} \int K_{\sigma \sigma^{\prime}}\left(\mathbf{r}, \mathbf{r}^{\prime}\right) \delta \rho_{\sigma^{\prime}}\left(\mathbf{r}^{\prime}, \omega\right) d \mathbf{r}^{\prime},
$$

where, the second term on the right-hand side (RHS) is the field-induced potential, $\sigma$ is the electron spin $(\sigma=\uparrow$ for 
spin-up or $\sigma=\downarrow$ for spin-down), $\delta \rho_{\sigma}(\mathbf{r}, \omega)$ is the fieldinduced electron density which is the deviation of the perturbed electron density from the unperturbed electron density $\rho_{\sigma}(\mathbf{r})$, and $K_{\sigma \sigma^{\prime}}\left(\mathbf{r}, \mathbf{r}^{\prime}\right)$ is the kernel function

$$
K_{\sigma \sigma^{\prime}}\left(\mathbf{r}, \mathbf{r}^{\prime}\right)=\frac{1}{\left|\mathbf{r}-\mathbf{r}^{\prime}\right|}+\frac{\delta V_{x c \sigma}(\mathbf{r})}{\delta \rho_{\sigma^{\prime}}\left(\mathbf{r}^{\prime}\right)}
$$

Here, the first and second terms on the RHS represent the field-induced changes of Hartree potential and XC potential $V_{x c \sigma}(\mathbf{r})$, respectively.

The field-induced electron density $\delta \rho_{\sigma}(\mathbf{r}, \omega)$ is related to the total perturbing potential $\phi_{\sigma}^{S C F}$ by

$$
\delta \rho_{\sigma}(\mathbf{r}, \omega)=\int \chi_{\sigma}\left(\mathbf{r}, \mathbf{r}^{\prime}, \omega\right) \phi_{\sigma}^{S C F}\left(\mathbf{r}^{\prime}, \omega\right) d \mathbf{r}^{\prime},
$$

where, $\chi_{\sigma}$ is the complex susceptibility given by

$$
\begin{aligned}
\chi_{\sigma}\left(\mathbf{r}, \mathbf{r}^{\prime}, \omega\right)= & \sum_{i} \sum_{j}^{\text {all }}\left[\frac{\gamma_{i \sigma}\left(\mathbf{r}, \mathbf{r}^{\prime}\right) \gamma_{j \sigma}^{*}\left(\mathbf{r}, \mathbf{r}^{\prime}\right)}{\hbar \omega-\left(\varepsilon_{j \sigma}-\varepsilon_{i \sigma}\right)+i \epsilon}\right. \\
& \left.-\frac{\gamma_{i \sigma}^{*}\left(\mathbf{r}, \mathbf{r}^{\prime}\right) \gamma_{j \sigma}\left(\mathbf{r}, \mathbf{r}^{\prime}\right)}{\hbar \omega+\left(\varepsilon_{j \sigma}-\varepsilon_{i \sigma}\right)+i \epsilon}\right] .
\end{aligned}
$$

Here, $\gamma_{i \sigma}\left(\mathbf{r}, \mathbf{r}^{\prime}\right)=\varphi_{i \sigma}^{*}(\mathbf{r}) \varphi_{i \sigma}\left(\mathbf{r}^{\prime}\right), \varphi_{i \sigma}(\mathbf{r})$ is the $i$ th electron spinorbital, $\epsilon$ is a positive infinitesimal, and the notations all and occ represent that the sums run over all and occupied electron orbitals, respectively.

From Eqs. (1) and (3), $\phi_{\sigma}^{S C F}(\mathbf{r}, \omega)$ and $\delta \rho_{\sigma}(\mathbf{r}, \omega)$ have to be calculated in a self-consistent field procedure. Applying Eq. (1) to Eq. (3) and introducing a kernel function

$$
N_{\sigma \sigma^{\prime}}\left(\mathbf{r}, \mathbf{r}^{\prime}, \omega\right)=\int \chi_{\sigma}\left(\mathbf{r}, \mathbf{r}^{\prime \prime}, \omega\right) K_{\sigma \sigma^{\prime}}\left(\mathbf{r}^{\prime \prime}, \mathbf{r}^{\prime}\right) d \mathbf{r}^{\prime \prime},
$$

one obtains an equation for $\delta \rho_{\sigma}(\mathbf{r}, \omega)$

$$
\begin{gathered}
\sum_{\sigma^{\prime}} \int\left[\delta_{\sigma \sigma^{\prime}} \delta\left(\mathbf{r}-\mathbf{r}^{\prime}\right)-N_{\sigma \sigma^{\prime}}\left(\mathbf{r}, \mathbf{r}^{\prime}, \omega\right)\right] \delta \rho_{\sigma^{\prime}}\left(\mathbf{r}^{\prime}, \omega\right) d \mathbf{r}^{\prime} \\
=\int \chi_{\sigma}\left(\mathbf{r}, \mathbf{r}^{\prime}, \omega\right) \phi^{e x t}\left(\mathbf{r}^{\prime}, \omega\right) d \mathbf{r}^{\prime} .
\end{gathered}
$$

The polarizability $\alpha(\omega)$ is the ratio of the induced dipole moment to the external field strength

$$
\begin{aligned}
\alpha(\omega) & =-\frac{1}{\mathcal{E}_{0}} \sum_{\sigma} \int z \delta \rho_{\sigma}(\mathbf{r}, \omega) d \mathbf{r} \\
& =-\frac{1}{\mathcal{E}_{0}^{2}} \sum_{\sigma} \iint \phi^{e x t}(\mathbf{r}, \omega) \chi_{\sigma}\left(\mathbf{r}, \mathbf{r}^{\prime}, \omega\right) \phi_{\sigma}^{S C F}\left(\mathbf{r}^{\prime}, \omega\right) d \mathbf{r}^{\prime} d \mathbf{r}
\end{aligned}
$$

The PICS $\sigma(\omega)$ is calculated by

$$
\sigma(\omega)=\frac{4 \pi \omega}{c} \operatorname{Im} \alpha(\omega),
$$

where $c$ is the speed of light.

The LRA is usually referred to as a time-dependent method since it takes the time-dependent field-induced elec- tron density into account. In contrast, if the field-induced electron density is neglected in Eq. (1), one has $\phi_{\sigma}^{S C F}=\phi_{\sigma}^{e x t}$. In this case, the cross section Eq. (7) is reduced to the result of independent-particle approximation, which is referred to as a time-independent method.

\section{B. Electron spin-orbitals}

To calculate the occupied electron spin-orbitals we use the SLHF density-functional approach $[21,23,24]$. In this approach, the electron spin-orbital $\varphi_{i \sigma}(\mathbf{r})$ and orbital energy $\varepsilon_{i \sigma}$ are calculated from the KS equation

$$
\left[-\frac{1}{2} \nabla^{2}+V_{\sigma}^{\mathrm{eff}}(\mathbf{r})\right] \varphi_{i \sigma}(\mathbf{r})=\varepsilon_{i \sigma} \varphi_{i \sigma}(\mathbf{r}),
$$

where, $V_{\sigma}^{\text {eff }}$ is the local effective potential given by

$$
V_{\sigma}^{\mathrm{eff}}(\mathbf{r})=-\frac{Z}{r}+\sum_{\sigma} \int \frac{\rho_{\sigma}\left(\mathbf{r}^{\prime}\right)}{\left|\mathbf{r}-\mathbf{r}^{\prime}\right|} d \mathbf{r}^{\prime}+V_{x c \sigma}(\mathbf{r}) .
$$

In Eq. (10), the spin-dependent electron density is calculated by $\rho_{\sigma}(\mathbf{r})=\sum_{i=1}^{N_{\sigma}}\left|\varphi_{i \sigma}(\mathbf{r})\right|^{2}$, where $N_{\sigma}$ is the number of electron with spin $\sigma$. On the RHS of Eq. (10), the first term is Coulomb potential, the second term is Hartree potential, and the third term $V_{x c \sigma}(\mathbf{r})$ is the XC potential. The XC potential can be decomposed into exchange potential $V_{x \sigma}(\mathbf{r})$ and correlation potential $V_{c \sigma}(\mathbf{r})$. The SLHF exchange potential $V_{x \sigma}^{\mathrm{SLHF}}(\mathbf{r})$ is given by $[21,23,24,26]$

$$
\begin{aligned}
V_{x \sigma}^{\mathrm{SLHF}}(\mathbf{r})= & -\frac{1}{\rho_{\sigma}(\mathbf{r})} \int \frac{\gamma_{\sigma}\left(\mathbf{r}, \mathbf{r}^{\prime}\right) \gamma_{\sigma}\left(\mathbf{r}^{\prime}, \mathbf{r}\right)}{\left|\mathbf{r}-\mathbf{r}^{\prime}\right|} d \mathbf{r}^{\prime} \\
& +\frac{1}{\rho_{\sigma}(\mathbf{r})} \int \gamma_{\sigma}\left(\mathbf{r}, \mathbf{r}^{\prime}\right) V_{x \sigma}^{\mathrm{SLHF}}\left(\mathbf{r}^{\prime}\right) \gamma_{\sigma}\left(\mathbf{r}^{\prime}, \mathbf{r}\right) d \mathbf{r}^{\prime} \\
& +\frac{1}{\rho_{\sigma}(\mathbf{r})} \iint \frac{\gamma_{\sigma}\left(\mathbf{r}, \mathbf{r}^{\prime}\right) \gamma_{\sigma}\left(\mathbf{r}^{\prime}, \mathbf{r}^{\prime \prime}\right) \gamma_{\sigma}\left(\mathbf{r}^{\prime \prime}, \mathbf{r}\right)}{\left|\mathbf{r}^{\prime}-\mathbf{r}^{\prime \prime}\right|} d \mathbf{r}^{\prime} d \mathbf{r}^{\prime \prime},
\end{aligned}
$$

where, $\gamma_{\sigma}\left(\mathbf{r}, \mathbf{r}^{\prime}\right)=\sum_{i=1}^{N_{\sigma}} \gamma_{i \sigma}\left(\mathbf{r}, \mathbf{r}^{\prime}\right)$. On the RHS of Eq. (11), the first term is Slater potential [22] and the second and third terms are the corrections to the Slater potential. The $V_{x \sigma}^{\mathrm{SLHF}}(\mathbf{r})$ behaves asymptotically as Slater potential and thus approaches to the correct $-1 / r$ at long range [26]. As for the correlation effect, the second-order gradient correlation potential and energy functional proposed by Lee, Yang, and Parr (LYP) [27] can provide an excellent correlation energy for atomic systems and has been incorporated into the calculation to estimate the correlation effect in this work.

\section{Green's function}

In Eq. (4) the sum over $j$ needs all the (occupied and unoccupied bound and continuum) electron orbitals. This makes it extremely difficult to accurately calculate $\chi_{\sigma}\left(\mathbf{r}, \mathbf{r}^{\prime}, \omega\right)$ directly from Eq. (4). To circumvent this difficulty, a Green's function associated with the KS equation has been introduced to calculate $\chi_{\sigma}[3,6,8]$. The Green's function $G_{\sigma}\left(\mathbf{r}, \mathbf{r}^{\prime}, E\right)$ is calculated by 


$$
\left[E+\frac{1}{2} \nabla^{2}-V_{\sigma}^{\mathrm{eff}}(\mathbf{r})\right] G_{\sigma}\left(\mathbf{r}, \mathbf{r}^{\prime}, E\right)=\delta\left(\mathbf{r}-\mathbf{r}^{\prime}\right),
$$

under appropriate boundary conditions. The Green's function can be expanded in terms of a complete set of KS electron spin-orbitals as [3]

$$
G_{\sigma}\left(\mathbf{r}, \mathbf{r}^{\prime}, E\right)=\sum_{j}^{\text {all }} \frac{\gamma_{j \sigma}^{*}\left(\mathbf{r}, \mathbf{r}^{\prime}\right)}{E-\varepsilon_{j \sigma} \pm i \epsilon}
$$

Applying Eq. (13) to Eq. (4) one has

$$
\begin{aligned}
\chi_{\sigma}\left(\mathbf{r}, \mathbf{r}^{\prime}, \omega\right)= & \sum_{i}^{o c c}\left[\gamma_{i \sigma}\left(\mathbf{r}, \mathbf{r}^{\prime}\right) G_{\sigma}\left(\mathbf{r}, \mathbf{r}^{\prime}, \varepsilon_{i \sigma}+\hbar \omega\right)\right. \\
& \left.+\gamma_{i \sigma}^{*}\left(\mathbf{r}, \mathbf{r}^{\prime}\right) G_{\sigma}^{*}\left(\mathbf{r}, \mathbf{r}^{\prime}, \varepsilon_{i \sigma}-\hbar \omega\right)\right] .
\end{aligned}
$$

Thus with assistance of the Green's function, only the occupied orbitals are needed to compute $\chi_{\sigma}$.

\section{COMPUTATIONAL DETAILS}

\section{A. Electron spin-orbitals}

The electron spin-orbitals of an atomic system can be calculated by using the procedure previously developed in Refs. $[21,24]$. In spherical coordinates, the electron spin-orbital $\varphi_{i \sigma}(\mathbf{r})$ is expressed as a product of a radial spin-orbital $R_{n l \sigma}(r)$ and a spherical harmonic $Y_{l m}(\theta, \phi)$,

$$
\varphi_{i \sigma}(\mathbf{r})=\frac{R_{n l \sigma}(r)}{r} Y_{l m}(\theta, \phi),
$$

where $n$ is the principal quantum number, $l$ is the orbital angular momentum quantum number, $m$ is the azimuthal quantum number, and $i$ is a set of quantum numbers except the spin $\sigma$. The radial spin-orbital $R_{n l \sigma}(r)$ is calculated from the radial $\mathrm{KS}$ equation $[21,24]$

$$
\left[-\frac{1}{2} \frac{d^{2}}{d r^{2}}+\frac{l(l+1)}{2 r^{2}}+v_{\sigma}^{\mathrm{eff}}(r)\right] R_{n l \sigma}=\varepsilon_{n l \sigma} R_{n l \sigma},
$$

where, $v_{\sigma}^{\text {eff }}(r)$ is the radial effective potential [21]. To obtain high-precision electron spin-orbital and orbital energy, we use generalized pseudospectral (GPS) method [28] to discretize the radial KS Eq. (16). The GPS method associated with an appropriate mapping technique can overcome difficulties due to singularity at $r=0$ and long-tail at large $r$ of the Coulomb interaction. It allows for nonuniform and optimal spatial discretization with the use of only a modest number of grid points. It has been shown that the GPS method is a very effective and efficient numerical algorithm for the highprecision solution of KS equation [21,24,29,30].

\section{B. Green's function}

In spherical coordinates, the Green's function can be expanded in terms of partial waves as

$$
G_{\sigma}\left(\mathbf{r}, \mathbf{r}^{\prime}, E\right)=\sum_{L M} Y_{L M}^{*}(\theta, \varphi) \mathcal{G}_{L \sigma}\left(r, r^{\prime}, E\right) Y_{L M}\left(\theta^{\prime}, \varphi^{\prime}\right),
$$

where, $\mathcal{G}_{L \sigma}\left(r, r^{\prime}, E\right)$ is the radial Green's function, which, with the appropriate boundary conditions, is determined by an inhomogeneous equation $[3,11]$

$$
\begin{aligned}
{[E} & \left.+\frac{1}{2} \frac{1}{r^{2}} \frac{\partial}{\partial r} r^{2} \frac{\partial}{\partial r}-\frac{1}{2} \frac{L(L+1)}{r^{2}}-v_{\sigma}^{\mathrm{eff}}(r)\right] \mathcal{G}_{L \sigma}\left(r, r^{\prime}, E\right) \\
& =\frac{\delta\left(r-r^{\prime}\right)}{r^{2}} .
\end{aligned}
$$

Alternatively, the radial Green's function can also be constructed from the solutions of a homogeneous equation

$$
\left[-\frac{d^{2}}{d r^{2}}+\frac{L(L+1)}{r^{2}}+2 v_{\sigma}^{\text {eff }}(r)-k^{2}\right] u_{L k \sigma}(r)=0,
$$

where, $k=\sqrt{2 E}$. If $\phi_{L k \sigma}(r)$ is the solution of Eq. (19) being regular at the origin and $\psi_{L k \sigma}(r)$ the solution behaving asymptotically as $r h_{L}^{(1)}(k r)$ (where $h_{L}^{(1)}$ is the spherical Hankel function of the first kind), the radial Green's function can be calculated by [11]

$$
\mathcal{G}_{L \sigma}\left(r, r^{\prime}, E\right)=\frac{2}{W} \frac{\phi_{L k \sigma}\left(r_{<}\right) \psi_{L k \sigma}\left(r_{>}\right)}{r r^{\prime}},
$$

where, $r_{<}\left(r_{>}\right)$refers to the smaller (larger) of $r$ and $r^{\prime}$ and $W=\phi_{L k \sigma} \psi_{L k \sigma}^{\prime}-\phi_{L k \sigma}^{\prime} \psi_{L k \sigma}$ is the Wronskian of $\phi_{L k \sigma}$ and $\psi_{L k \sigma}$.

\section{Absorber}

In principle, the boundary conditions at $r \rightarrow \infty$ is required to calculate $\psi_{L k \sigma}(r)$. In reality, the boundary cannot be set at $r \rightarrow \infty$. No matter how far the boundary is, as long as it is located at finite distance, the outgoing wave function $\psi_{L k \sigma}(r)$ with $E>0$ may reflect on the boundary, making the PICS oscillating artificially. To remove the reflection we introduce an absorber for each outgoing wave function. The absorber is characterized by an absorptive potential with a linear dependence of the radial coordinate

$$
U(r)=\left\{\begin{array}{ll}
0, & 0 \leq r<r_{a} \\
-U_{0} \frac{r-r_{a}}{r_{\max }-r_{a}}, & r_{a} \leq r \leq r_{\max }
\end{array},\right.
$$

where $r_{\max }$ is the radial coordinate of the boundary; $U_{0}$ and $r_{a}$ are two parameters representing the strength and starting position of the absorber, respectively. Obviously, $r_{\max }-r_{a}$ represents the width of the absorber. Similar absorbers have been used in the wave packet method of molecular collisions $[31,32]$ and photoionization of molecules and atomic clusters recently [33].

When taking the absorptive potential into account the outgoing wave function is calculated from an equation obtained by replacing $v_{\sigma}^{\text {eff }}(r)$ with $v_{\sigma}^{\text {eff }}(r)+i U(r)$ in Eq. (19). Since the behavior of an outgoing wave function depends on electron spin-orbitals through both $v_{\sigma}^{\text {eff }}(r)$ and $k(E)$ the absorber parameters may be different for different electron spin-orbitals. 


\section{Susceptibility and cross sections}

In spherical coordinates, the susceptibility $\chi_{\sigma}\left(\mathbf{r}, \mathbf{r}^{\prime}, \omega\right)$ can also be expanded in the partial waves [11]

$$
\chi_{\sigma}\left(\mathbf{r}, \mathbf{r}^{\prime}, \omega\right)=\sum_{l m} Y_{l m}^{*}(\theta, \phi) \chi_{l \sigma}\left(r, r^{\prime}, \omega\right) Y_{l m}\left(\theta^{\prime}, \phi^{\prime}\right) .
$$

From Eqs. (14), (15), (17), and (22), the partial wave susceptibility $\chi_{l \sigma}\left(r, r^{\prime}, \omega\right)$ is calculated by

$$
\begin{aligned}
\chi_{l \sigma}\left(r, r^{\prime}, \omega\right)= & \frac{1}{4 \pi} \sum_{n^{\prime} l^{\prime}} \sum_{L} w_{n^{\prime} l^{\prime} \sigma} \frac{R_{n^{\prime} l^{\prime} \sigma}(r)}{r} \frac{R_{n^{\prime} l^{\prime} \sigma}\left(r^{\prime}\right)}{r^{\prime}}\left|\left\langle l 0 l^{\prime} 0 \mid L 0\right\rangle\right|^{2} \\
& \times\left[\mathcal{G}_{L \sigma}\left(r, r^{\prime}, \varepsilon_{n^{\prime} l^{\prime} \sigma}+\hbar \omega\right)\right. \\
& \left.+\mathcal{G}_{L \sigma}^{*}\left(r, r^{\prime}, \varepsilon_{n^{\prime} l^{\prime} \sigma}-\hbar \omega\right)\right] .
\end{aligned}
$$

Furthermore, expanding $\delta \rho_{\sigma}(\mathbf{r}, \omega)$ in the partial waves $\delta \rho_{\sigma}(\mathbf{r}, \omega)=\Sigma_{l m} \delta \rho_{l m \sigma}(r, \omega) Y_{l m}(\theta, \phi)$, using $z=\sqrt{\frac{4 \pi}{3}} r Y_{10}(\theta, \phi)$, and from Eq. (6), we obtain

$$
\begin{aligned}
\delta \rho_{l m \sigma}(r, \omega)= & \mathcal{E}_{0} \sqrt{\frac{4 \pi}{3}} \int \chi_{1 \sigma}\left(r, r^{\prime}, \omega\right) r^{\prime 3} d r^{\prime} \delta_{l 1} \delta_{m 0} \\
& +\sum_{\sigma^{\prime}} \int N_{l \sigma \sigma^{\prime}}\left(r, r^{\prime}, \omega\right) \delta \rho_{l m \sigma^{\prime}}\left(r^{\prime}, \omega\right) r^{\prime 2} d r^{\prime},
\end{aligned}
$$

where $N_{l \sigma \sigma^{\prime}}\left(r, r^{\prime}, \omega\right)$ is the partial wave component in the partial wave expansion $N_{\sigma \sigma^{\prime}}\left(\mathbf{r}, \mathbf{r}^{\prime}\right)$ $=\Sigma_{l m} Y_{l m}^{*}(\theta, \phi) N_{l \sigma \sigma^{\prime}}\left(r, r^{\prime}, \omega\right) Y_{l m}\left(\theta^{\prime}, \phi^{\prime}\right)$. Solving Eq. (24) one obtains the partial wave component $\delta \rho_{l m \sigma}(r, \omega)$. From Eq. (7), the polarizability $\alpha(\omega)$ is given by

$$
\alpha(\omega)=-\frac{1}{\mathcal{E}_{0}} \sqrt{\frac{4 \pi}{3}} \sum_{\sigma} \int \delta \rho_{10 \sigma}(r, \omega) r^{3} d r .
$$

The PICS $\sigma(\omega)$ is calculated from Eq. (8) by using $\alpha(\omega)$.

\section{RESULTS AND DISCUSSION}

\section{A. Photoionization from the ground state of $\mathrm{Ne}$}

To test the approach developed in the preceding sections we first apply it to the calculation of PICS from the ground state of Ne. The total PICS is plotted in Fig. 1, where the solid and dashed lines are the results of time-dependent spindependent localized Hartree-Fock (TDSLHF) and timeindependent spin-dependent localized Hartree-Fock (TISLHF) methods, respectively. Also plotted in this figure are recent experimental data $[34,35]$ for comparison. It is shown that the TISLHF PICS are substantially larger than the experimental results in a broad range of lower photon energy. The maximum deviations to the experimental results in Ref. [34] and Ref. [35] are 35.9\% and 31.3\% before the resonance region and $33.0 \%$ and $24.6 \%$ after the resonance region, respectively. The TDSLHF cross sections, however, are much closer to the experimental results and show significant improvement in spite of the underestimate near the ionization threshold (IT) of $2 p$ electron. Apart from the energy region adjacent to the IT of $2 p$ electron where the maximum devia-

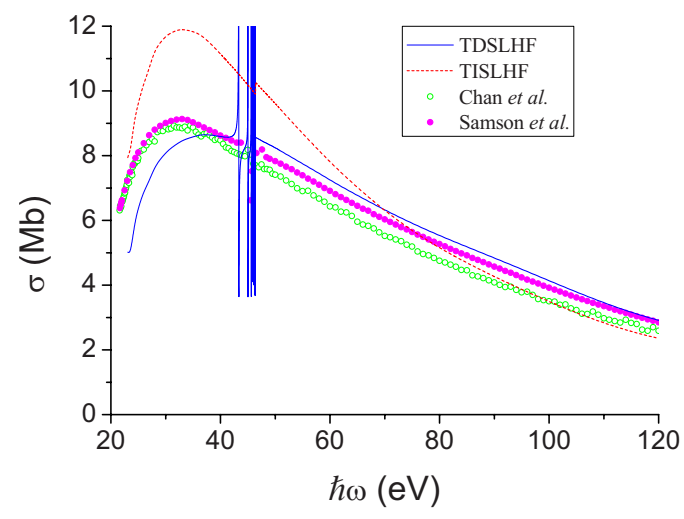

FIG. 1. (Color online) Total photoionization cross sections from the ground state of Ne: the solid and dashed lines are the results of TDSLHF and TISLHF methods, respectively. The open and solid circles are the experimental data of Chan et al. [34] and Samson et al. [35].

tions are $31.0 \%$ and $32.4 \%$, the discrepancies to the experimental results in Ref. [34] and Ref. [35] are not more than $20.0 \%$ and $20.0 \%$ before the resonance region and less than $19.9 \%$ and $6.1 \%$ after the resonance region, respectively. In the range of photon energy shown in Fig. 1, except a small stepwise enhancement due to the contribution of $2 s$ electron photoionization at the IT energy of $2 s$ electron $46.405 \mathrm{eV}$, the TISLHF PICS are structureless and decrease monotonically above the IT of $2 s$ electron. The TDSLHF PICS, however, appear a series of sharp resonances in the energy region from
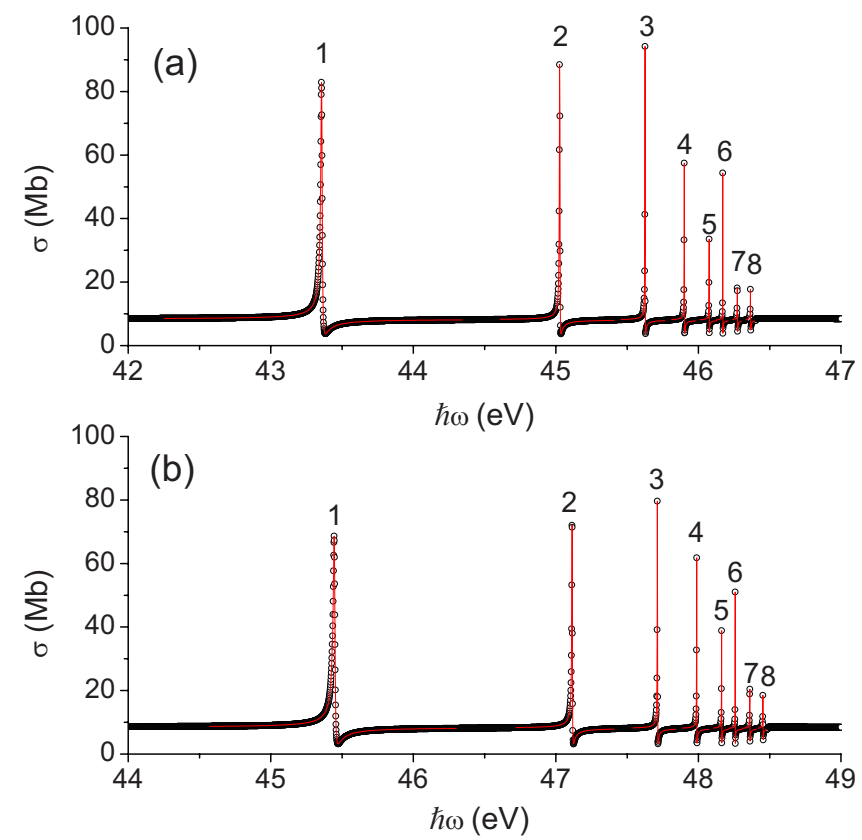

FIG. 2. (Color online) Autoionization resonances in the tota photoionization cross sections near the ionization threshold of $2 p$ electron: (a) The results of TDSLHF method and (b) the results of TDSLHF method with the $2 s$ electron orbital energy being replaced by the experimental value. The resonance peaks 1 to 8 are produced by the transitions $2 s \rightarrow n p$ with $n=3$ to 10 , respectively. The open circles are the numerical results and the solid lines are the fitted results to the Fano profile. 
TABLE I. Profile parameters of the autoionization resonances produced by the transitions from the ground state of Ne to the Rydberg series $1 s^{2} 2 s 2 p^{6} n p$ with $n=3$ to 8 .

\begin{tabular}{|c|c|c|c|c|c|c|}
\hline Resonant transition & Approach & $\begin{array}{c}\sigma_{0} \\
(\mathrm{Mb})\end{array}$ & $\begin{array}{c}\Gamma \\
(\mathrm{meV})\end{array}$ & $q$ & $\eta^{2}$ & $\begin{array}{c}E_{r} \\
(\mathrm{eV})\end{array}$ \\
\hline \multirow[t]{7}{*}{$2 s \rightarrow 3 p$} & TDSLHF $^{\mathrm{a}}$ & 8.34 & 12.80 & -4.03 & 0.551 & 43.358 \\
\hline & S-TDSLHF ${ }^{b}$ & 8.40 & 15.23 & -3.40 & 0.619 & 45.447 \\
\hline & $\mathrm{S}-\mathrm{EXX}+\mathrm{ALDA}^{\mathrm{c}}$ & 8.09 & 17.9 & -3.18 & 0.547 & 45.438 \\
\hline & S-TDLSDA ${ }^{\mathrm{d}}$ & 8.28 & 18.04 & -2.40 & 0.764 & 45.453 \\
\hline & TDLDA $^{\mathrm{e}}$ & 8.18 & 13.90 & -3.69 & 0.514 & 46.253 \\
\hline & $R$ matrix $^{\mathrm{f}}$ & & 34.9 & & & 45.534 \\
\hline & Expt. $^{\mathrm{g}}$ & $8.6 \pm 0.6$ & $13 \pm 2$ & $-1.6 \pm 0.2$ & $0.70 \pm 0.07$ & $45.546 \pm 0.008$ \\
\hline \multirow[t]{7}{*}{$2 s \rightarrow 4 p$} & TDSLHF $^{\mathrm{a}}$ & 8.12 & 3.89 & -4.35 & 0.543 & 45.028 \\
\hline & S-TDSLHF ${ }^{b}$ & 8.15 & 4.55 & -3.74 & 0.606 & 47.115 \\
\hline & $\mathrm{S}-\mathrm{EXX}+\mathrm{ALDA}^{\mathrm{c}}$ & 7.89 & 5.5 & -3.35 & 0.528 & 47.093 \\
\hline & S-TDLSDA $^{\mathrm{d}}$ & 8.06 & 5.14 & -2.62 & 0.783 & 47.098 \\
\hline & TDLDA $^{\mathrm{e}}$ & 7.98 & 3.86 & -3.95 & 0.505 & 47.397 \\
\hline & $R$ matrix $^{\mathrm{f}}$ & & 6.65 & & & 47.111 \\
\hline & Expt. $^{\mathrm{g}}$ & $8.0 \pm 0.6$ & $4.5 \pm 1.5$ & $-1.6 \pm 0.3$ & $0.70 \pm 0.07$ & $47.121 \pm 0.005$ \\
\hline \multirow[t]{6}{*}{$2 s \rightarrow 5 p$} & TDSLHF $^{\mathrm{a}}$ & 8.05 & 1.55 & -4.47 & 0.537 & 45.625 \\
\hline & S-TDSLHF ${ }^{b}$ & 8.06 & 1.81 & -3.85 & 0.600 & 47.713 \\
\hline & S-TDLSDA $^{\mathrm{d}}$ & 7.91 & 2.20 & -2.72 & 0.783 & 47.683 \\
\hline & TDLDA $^{\mathrm{e}}$ & 7.91 & 1.62 & -4.05 & 0.502 & 47.814 \\
\hline & $R$ matrix $^{\mathrm{f}}$ & & 2.47 & & & 47.692 \\
\hline & Expt. $^{\mathrm{g}}$ & $8.2 \pm 0.6$ & $2 \pm 1$ & $-1.6 \pm 0.5$ & $0.70 \pm 0.14$ & $47.692 \pm 0.005$ \\
\hline \multirow[t]{4}{*}{$2 s \rightarrow 6 p$} & TDSLHF $^{\mathrm{a}}$ & 8.00 & 0.89 & -4.57 & 0.539 & 45.901 \\
\hline & S-TDSLHF ${ }^{\mathrm{b}}$ & 8.00 & 1.04 & -3.94 & 0.601 & 47.989 \\
\hline & $R$ matrix $^{\mathrm{f}}$ & & 1.28 & & & 47.967 \\
\hline & Expt. $^{\mathrm{g}}$ & & & & & $47.967 \pm 0.006$ \\
\hline \multirow[t]{4}{*}{$2 s \rightarrow 7 p$} & TDSLHF $^{\mathrm{a}}$ & 7.99 & 0.43 & -4.99 & 0.555 & 46.075 \\
\hline & S-TDSLHF ${ }^{b}$ & 8.01 & 0.59 & -3.88 & 0.585 & 48.163 \\
\hline & $R$ matrix $^{\mathrm{f}}$ & & 0.73 & & & 48.119 \\
\hline & Expt. $^{\mathrm{g}}$ & & & & & $48.116 \pm 0.006$ \\
\hline \multirow[t]{4}{*}{$2 s \rightarrow 8 p$} & TDSLHF $^{\mathrm{a}}$ & 7.94 & 0.37 & -4.54 & 0.534 & 46.171 \\
\hline & S-TDSLHF ${ }^{b}$ & 7.96 & 0.42 & -3.92 & 0.605 & 48.258 \\
\hline & $R$ matrix ${ }^{\mathrm{f}}$ & & 0.46 & & & 48.211 \\
\hline & Expt. $^{\mathrm{g}}$ & & & & & $48.207 \pm 0.006$ \\
\hline
\end{tabular}

${ }^{\mathrm{a}}$ TDSLHF results.

${ }^{\mathrm{b}}$ TDSLHF results with the experimental $2 s$ electron orbital energy.

${ }^{c} \mathrm{EXX}+\mathrm{ALDA}$ results with the experimental $2 s$ electron orbital energy [10].

${ }^{\mathrm{d}}$ TDLSDA results with the experimental $2 s$ electron orbital energy [6].

TDLDA results [7].

${ }^{\mathrm{f}} R$-matrix results [38].

${ }^{\mathrm{g}}$ Experimental results [37].

43.0 to $46.4 \mathrm{eV}$. In the higher energy region, both TDSLHF and TISLHF results are getting closer and approach to the experimental results.
The sharp resonances in the PICS are produced by the resonant transitions $2 s \rightarrow n p$ from the ground state $1 s^{2} 2 s^{2} 2 p^{6}$ to autoionization states $1 s^{2} 2 s 2 p^{6} n p$ with $n=3$ to 10 . In Fig. 

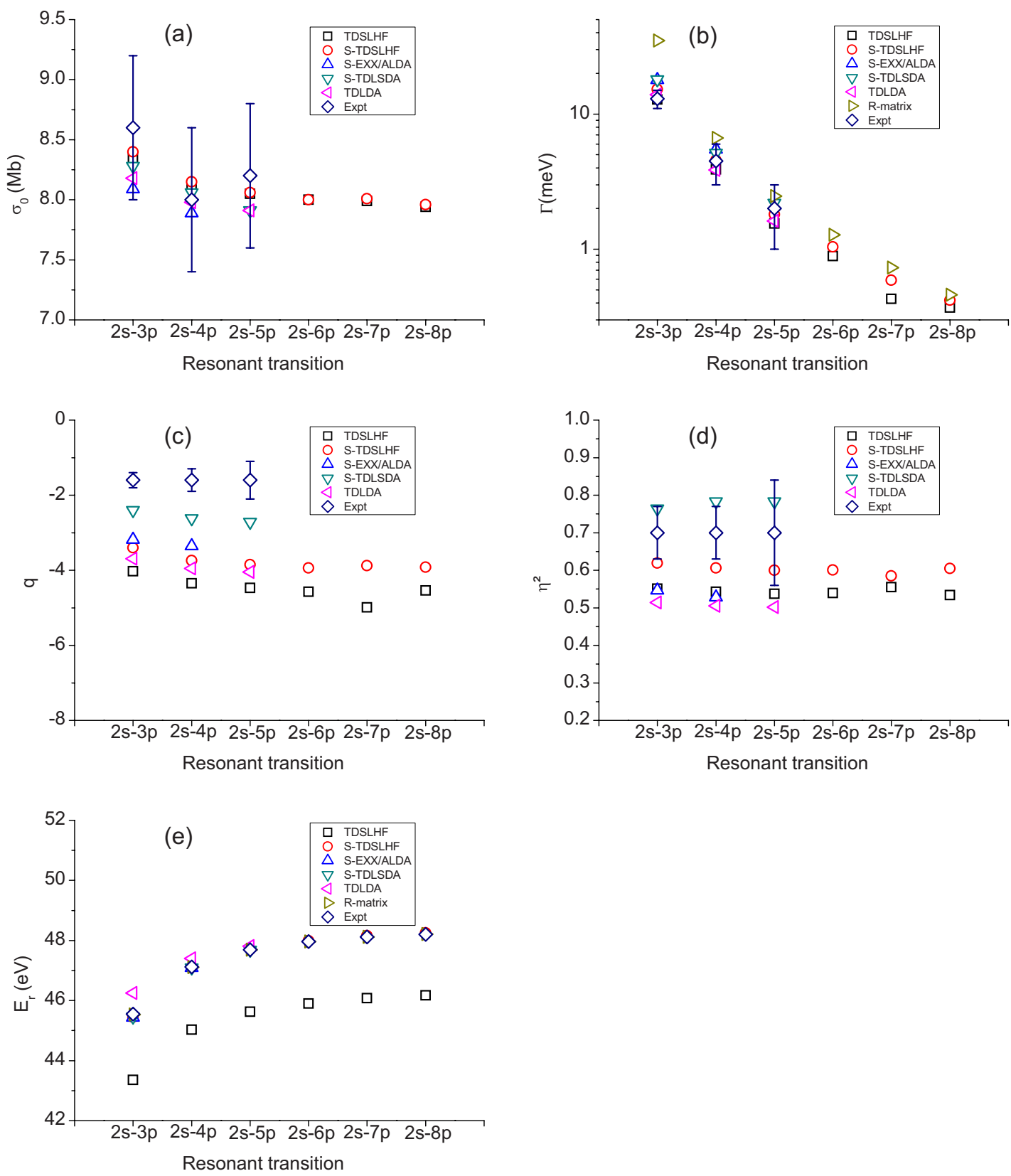

FIG. 3. (Color online) Profile parameters of autoionization resonances: (a) cross section $\sigma_{0}$, (b) linewidth $\Gamma$, (c) profile index $q$, (d) correlation coefficient $\eta^{2}$, and (e) resonance position $E_{r}$.

2(a) we show the details of the resonances in open circles. To quantitatively analyze the resonances and estimate the autoionization widths we fit the resonances to the Fano profile [36]

$$
\sigma(\omega)=\sigma_{0}\left[\eta^{2} \frac{(q+\kappa)^{2}}{1+\kappa^{2}}-\eta^{2}+1\right]
$$

where $\kappa=2\left(\omega-E_{r}\right) / \Gamma, E_{r}$ is the resonance position, $q$ is the profile index, $\Gamma$ is the linewidth of the resonance profile, $\eta^{2}$ is the correlation coefficient, and $\sigma_{0}$ is the cross section without correlation. In Fig. 2(a) we show the fitted resonances in the solid line. The fitted resonance profile parameters are given in Table I in the rows with TDSLHF and shown in Fig.
3 with open squares. Also listed in Table I and shown in Fig. 3 are the available experimental results [37] and the theoretical results of the time-dependent density-functional method employing exact exchange-only KS potential and adiabaticlocal-density-approximation exchange-correlation kernel with shifted $2 s$ orbital energy (S-EXX+ALDA) [10], timedependent local spin-density approximation with shifted $2 s$ orbital energy (S-TDLSDA) [6], time-dependent localdensity approximation (TDLDA) [7], and $R$-matrix method ( $R$ matrix) [38], for comparison. Figure 3(a) shows that for the cross section $\sigma_{0}$ the TDSLHF results are in good agreement with the experimental values and overall a little bit better than the other theoretical results. The deviations to the results of S-EXX+ALDA, S-TDLSDA, TDLDA, and ex- 


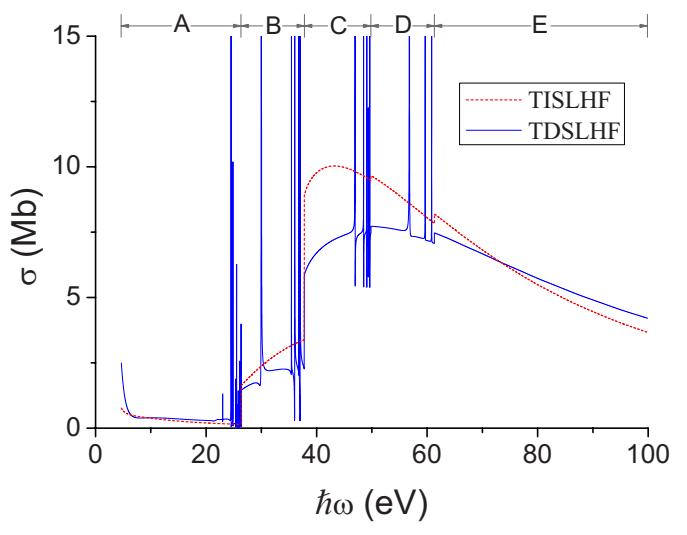

FIG. 4. (Color online) Total photoionization cross sections of $\mathrm{Ne}$ outer-shell excited state $1 s_{\downarrow} 1 s_{\uparrow} 2 s_{\downarrow} 2 s_{\uparrow} 2 p_{\downarrow}^{3} 2 p_{\uparrow}^{2} 3 s_{\uparrow}$ : the solid and dashed lines are the results of TDSLHF and TISLHF methods, respectively.

periments are less than $3.1 \%, 1.8 \%, 2.0 \%$, and $3.0 \%$, respectively. For the linewidth $\Gamma$ shown in Fig. 3(b) the TDSLHF results are close to the TDLDA results and better than those of S-EXX+ALDA, S-TDLSDA, and $R$ matrix compared to the experimental results. The maximum deviations of the results of TDSLHF, S-EXX+ALDA, S-TDLSDA, TDLDA, and $R$ matrix to the experimental results are $22.5 \%, 37.7 \%$, $38.7 \%, 19.0 \%$, and $168.5 \%$, respectively. For the line profile index $q$ in Fig. 3(c), all the theoretical results are much smaller than the experimental results and the TDSLHF results are close to those of TDLDA. The maximum discrepancies of the results of TDSLHF, S-EXX+ALDA, S-TDLSDA, and TDLDA to the experimental results are $179.4 \%, 109.4 \%, 70.0 \%$, and $153.1 \%$, respectively. For the correlation coefficient $\eta^{2}$ shown in Fig. 3(d), the TDSLHF results are very close to those of S-EXX+ALDA and TDLDA but smaller than the S-TDLSDA results and experimental data. The maximum discrepancies of the TDSLHF results to those of S-EXX+ALDA, S-TDLSDA, TDLDA, and experiments are $2.8 \%, 31.4 \%, 7.5 \%$, and $22.4 \%$, respectively. As for the resonance position $E_{r}$ in Fig. 3(e), all the resonances in TDSLHF PICS are about $2 \mathrm{eV}$ shift to the lower photon energy with respect to the experimental data. Similar phenomena were also found in Refs. $[6,10]$. This discrepancy mainly stems from the deviation of the $2 s$ electron orbital energy from the experimental value [6]. For demonstration, we notice that the $2 s$ electron orbital energy from the TDSLHF calculation is -1.707 a.u. which is about 2.04 $\mathrm{eV}$ higher than the experimental value -1.782 a.u. [39]. To explore influence of the orbital energy to the PICS and resonance profiles we have also performed a TDSLHF calculation with the $2 s$ electron orbital energy being replaced by the experimental value in the calculation of the Green's function. The calculated and fitted PICS in the resonance energy region are shown in Fig. 2(b) in the open circles and solid line, respectively. The fitted resonance profile parameters are listed in Table I in the rows with S-TDSLHF and shown in Fig. 3 with open circles. It is shown that with the $2 s$ electron orbital energy replaced by the experimental value the resonances shift to the higher photon energy about $2 \mathrm{eV}$ and the calculated resonance positions $E_{r}$ are in very good agreement with the experimental results. When using the theoretical value of the $2 s$ electron orbital energy, the maximum discrepancies of the TDSLHF results to those of S-EXX + ALDA, S-TDLSDA, TDLDA, $R$ matrix, and experiments are $4.6 \%, 4.6 \%, 6.3 \%, 4.8 \%$, and $4.8 \%$, respectively. While when using the experimental value of the $2 s$ electron orbital energy, the maximum deviations of the S-TDSLHF results to
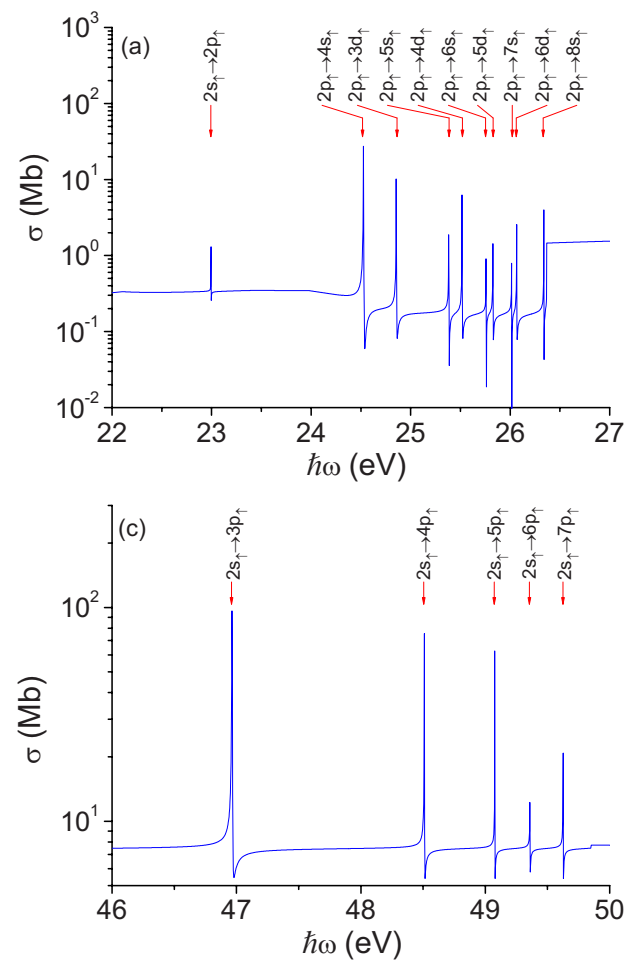
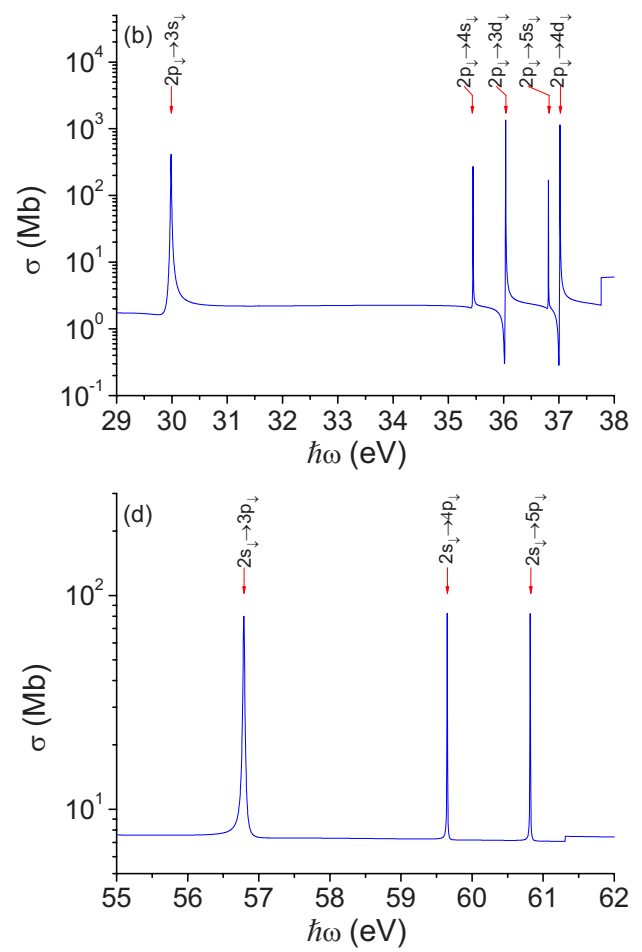

FIG. 5. (Color online) Autoionization resonances in the total photoionization cross sections of $\mathrm{Ne}$ outer-shell excited state $1 s_{\downarrow} 1 s_{\uparrow} 2 s_{\downarrow} 2 s_{\uparrow} 2 p_{\downarrow}^{3} 2 p_{\uparrow}^{2} 3 s_{\uparrow}$ : (a) The resonances produced by the transitions $2 p_{\uparrow} \rightarrow n s_{\uparrow}$ and $n^{\prime} d_{\uparrow}$ with $n$ $\geq 4$ and $n^{\prime} \geq 3$. Note that the resonance produced by the transition $2 s_{\uparrow} \rightarrow 2 p_{\uparrow}$ is embodied in this region. (b) The resonances produced by the transitions $2 p_{\downarrow} \rightarrow n s_{\downarrow}$ and $n d_{\downarrow}$ with $n \geq 3$. (c) The resonances produced by the transitions $2 s_{\uparrow}$ $\rightarrow n p_{\uparrow}$ with $n \geq 2$. Note that the resonance for $n=2$ is located in the energy region in (a). (d) The resonances produced by the transitions $2 s_{\downarrow} \rightarrow n p_{\downarrow}$ with $n \geq 3$. 
TABLE II. Autoionization resonance peak positions (in $\mathrm{eV}$ ) for the photoionizations from Ne outer-shell excited states $\left\{1 s_{\downarrow} 1 s_{\uparrow} 2 s_{\downarrow} 2 s_{\uparrow} 2 p_{\downarrow}^{3} 2 p_{\uparrow}^{2}\right\} n l_{\uparrow}$ with $n=3-4$ and $l=0-2$.

\begin{tabular}{|c|c|c|c|c|c|c|}
\hline \multirow[b]{2}{*}{ Resonant transition } & \multicolumn{6}{|c|}{ Initial states } \\
\hline & \{\}$^{\mathrm{a}} 3 \mathrm{~s}_{\uparrow}$ & \{\} $3 p_{\uparrow}$ & \{\} $3 d_{\uparrow}$ & \{\} $4 s_{\uparrow}$ & \{\} $4 p_{\uparrow}$ & \{\} $4 d_{\uparrow}$ \\
\hline $2 p_{\uparrow} \rightarrow 3 s_{\uparrow}$ & & 23.829 & 26.150 & 23.527 & 24.863 & 26.509 \\
\hline $4 s_{\uparrow}$ & 24.526 & 26.738 & 29.139 & & 27.693 & 29.554 \\
\hline $5 s_{\uparrow}$ & 25.383 & 27.630 & 30.153 & 26.839 & 28.581 & 30.452 \\
\hline $6 s_{\uparrow}$ & 25.759 & 28.014 & 30.575 & 27.206 & 28.972 & 30.896 \\
\hline $7 s_{\uparrow}$ & 26.013 & 28.221 & 30.803 & 27.405 & 29.175 & 31.119 \\
\hline $8 s_{\uparrow}$ & & 28.406 & 30.928 & 27.549 & 29.293 & 31.250 \\
\hline $9 s_{\uparrow}$ & & & 31.043 & & 29.386 & 31.334 \\
\hline $10 s_{\uparrow}$ & & & & & & 31.416 \\
\hline $3 d_{\uparrow}$ & 24.855 & 27.078 & 29.565 & 26.371 & 28.060 & 30.001 \\
\hline $4 d_{\uparrow}$ & 25.516 & 27.769 & 30.330 & 26.999 & 28.730 & 30.670 \\
\hline $5 d_{\uparrow}$ & 25.826 & 28.085 & 30.670 & 27.290 & 29.046 & 30.986 \\
\hline $6 d_{\uparrow}$ & 26.066 & 28.264 & 30.850 & 27.453 & 29.217 & 31.173 \\
\hline $7 d_{\uparrow}$ & & & 30.961 & 27.590 & 29.320 & 31.282 \\
\hline $8 d_{\uparrow}$ & & & & & 29.410 & 31.358 \\
\hline $2 p_{\downarrow} \rightarrow 3 s_{\downarrow}$ & 29.984 & 30.382 & 30.656 & 30.594 & 30.587 & 30.643 \\
\hline $4 s_{\downarrow}$ & 35.446 & 36.948 & 38.389 & 38.224 & 38.302 & 38.559 \\
\hline $5 s_{\downarrow}$ & 36.809 & 38.447 & 40.406 & 40.463 & 40.722 & 41.081 \\
\hline $6 s_{\downarrow}$ & & & & & 42.194 & 42.635 \\
\hline $3 d_{\downarrow}$ & 36.036 & 37.378 & 38.327 & 38.161 & 38.213 & 38.412 \\
\hline $4 d_{\downarrow}$ & 37.018 & 38.580 & 40.479 & 40.406 & 40.697 & 41.021 \\
\hline $5 d_{\downarrow}$ & & & & 41.693 & 41.995 & 42.423 \\
\hline $2 s_{\uparrow} \rightarrow 2 p_{\uparrow}$ & 22.994 & 23.032 & 23.032 & 23.048 & 23.081 & 23.056 \\
\hline $3 p_{\uparrow}$ & 46.964 & 49.187 & 51.685 & 48.719 & 50.243 & 52.129 \\
\hline $4 p_{\uparrow}$ & 48.510 & 50.798 & 53.310 & 50.044 & 51.767 & 53.682 \\
\hline $5 p_{\uparrow}$ & 49.075 & 51.381 & 53.960 & 50.572 & 52.352 & 54.265 \\
\hline $6 p_{\uparrow}$ & 49.359 & 51.659 & 54.260 & 50.833 & 52.630 & 54.577 \\
\hline $7 p_{\uparrow}$ & 49.623 & 51.835 & 54.420 & 50.986 & 52.782 & 54.745 \\
\hline $8 p_{\uparrow}$ & & 52.036 & 54.532 & 51.136 & 52.883 & 54.845 \\
\hline $9 p_{\uparrow}$ & & & 54.654 & & 52.978 & 54.923 \\
\hline $10 p_{\uparrow}$ & & & & & 53.098 & 55.013 \\
\hline $11 p_{\uparrow}$ & & & & & & 55.114 \\
\hline $2 s_{\downarrow} \rightarrow 3 p_{\downarrow}$ & 56.790 & 57.457 & 57.892 & 57.786 & 57.797 & 57.911 \\
\hline $4 p_{\downarrow}$ & 59.650 & 61.242 & 62.926 & 62.818 & 62.940 & 63.218 \\
\hline $5 p_{\downarrow}$ & 60.817 & 62.458 & 64.412 & 64.404 & 64.747 & 65.147 \\
\hline
\end{tabular}

$\overline{\bar{a}\{\}=1 s_{\downarrow} 1 s_{\uparrow} 2 s_{\downarrow} 2 s_{\uparrow} 2 p_{\downarrow}^{3} 2 p_{\uparrow}^{2} \text { is the abbreviation of the core electron configuration. }}$

those of S-EXX+ALDA, S-TDLSDA, TDLDA, $R$ matrix, and experiments are reduced to $0.05 \%, 0.06 \%, 1.74 \%$, $0.19 \%$, and $0.22 \%$, respectively. In addition, all the other resonance profile parameters, particularly $\Gamma$ and $\eta^{2}$, are also significantly improved and closer to the experimental values. The maximum deviations of the S-TDSLHF results to the experimental results are reduced to $2.3 \%, 17.2 \%, 140.6 \%$, and $13.4 \%$, respectively, for $\sigma_{0}, \Gamma, q$, and $\eta^{2}$.

\section{B. Photoionization from outer-shell excited states of $\mathrm{Ne}$}

For the photoionization from Ne excited states, particularly for those with autoionization resonances, both experi- 


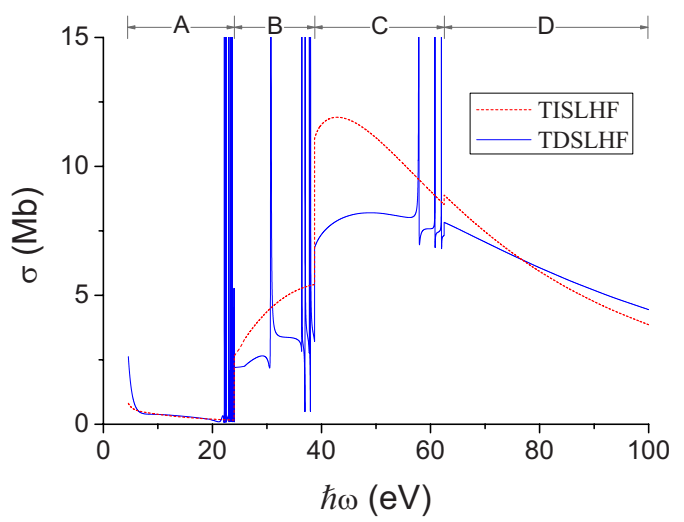

FIG. 6. (Color online) Total photoionization cross sections of $\mathrm{Ne}$ inner-shell excited state $1 s_{\uparrow} 1 s_{\downarrow} 2 s_{\uparrow} 2 p_{\uparrow}^{3} 2 p_{\downarrow}^{3} 3 s_{\downarrow}$ : the solid and dashed lines are the results of TDSLHF and TISLHF methods, respectively.

mental data [40,41] and theoretical results [41-45] are scarce. One of the advantages of the proposed approach is that it can be used to the calculation of the photoionization from excited states of atomic systems. Such kind of calculation can provide insight to interchannel interference and orbital relaxation in the photoionization processes from the excited states. We first apply the proposed approach to the computation of PICS of Ne outer-shell excited states. In Fig. 4, we show, in the solid and dashed lines, the total PICS of TDSLHF method and TISLHF method for the photoionization from the outer-shell excited state $1 s_{\downarrow} 1 s_{\uparrow} 2 s_{\downarrow} 2 s_{\uparrow} 2 p_{\downarrow}^{3} 2 p_{\uparrow}^{2} 3 s_{\uparrow}$ of $\mathrm{Ne}$, respectively. In Fig. 5 we show the details of autoionization resonances in the total PICS. It is shown that the total PICS can be divided into five regions A-E. They are separated by the steps at ITs in the TISLHF PICS. The first region A starts from the IT of $3 s_{\uparrow}$ electron and ends at the IT of $2 p_{\uparrow}$ electron followed by two series of sharp autoionization reso- nances in the TDSLHF PICS. These two series of resonances are produced by the transitions of a $2 p_{\uparrow}$ electron to the higher bound $s_{\uparrow}$ and $d_{\uparrow}$ orbitals, respectively, and thus converge to the IT of $2 p_{\uparrow}$ electron, as shown in Fig. 5(a). Unlike the PICS of the ground state which increase with photon energy near the IT of $2 p$ electron, the PICS of the excited state decrease with photon energy near the IT of the $3 s_{\uparrow}$ electron. The second region B starts from the IT of $2 p_{\uparrow}$ electron and ends at the IT of $2 p_{\downarrow}$ electron. In this region there are also two series of sharp resonances in the PICS, as shown in Fig. 5(b). They are induced by the transitions of a $2 p$, electron to the higher bound $s_{\downarrow}$ and $d_{\downarrow}$ orbitals, respectively, and converge to the IT of $2 p_{\downarrow}$ electron. The third region C starts from the IT of $2 p_{\downarrow}$ electron and ends at the IT of $2 s_{\uparrow}$ electron followed by a series of sharp resonances produced by the transitions of a $2 s_{\uparrow}$ electron to the higher bound $p_{\uparrow}$ orbitals, as shown in Fig. 5 (c). Note that one resonance produced by the transition $2 s_{\uparrow} \rightarrow 2 p_{\uparrow}$ in this series is located in the lower energy region A in Fig. 5(a). The fourth region D starts from the IT of $2 s_{\uparrow}$ electron and ends at the IT of $2 s_{\downarrow}$ electron. This region consists of a series of sharp resonances produced by the transitions of a $2 s_{\downarrow}$ electron to the higher bound $p_{\downarrow}$ orbitals as shown in Fig. 5(d). The last region E covers the energy region from the IT of $2 s_{\downarrow}$ electron to the maximum energy shown in Fig. 4. In this region the PICS are structureless since the next autoionization resonances occur at very high energy region when a $1 s_{\uparrow}$ electron is resonantly pumped to the higher bound $p_{\uparrow}$ orbitals. We have also performed the calculation of PICS from other outer-shell excited states of Ne. The total PICS have the similar structures as those from the excited state $1 s_{\downarrow} 1 s_{\uparrow} 2 s_{\downarrow} 2 s_{\uparrow} 2 p_{\downarrow}^{3} 2 p_{\uparrow}^{2} 3 s_{\uparrow}$ shown in Fig. 4 .

Interchannel interference has a significant impact on the autoionization resonances in the photoionization from excited states. Due to the interference of autoionization channels, the profiles of autoionization resonances, particularly
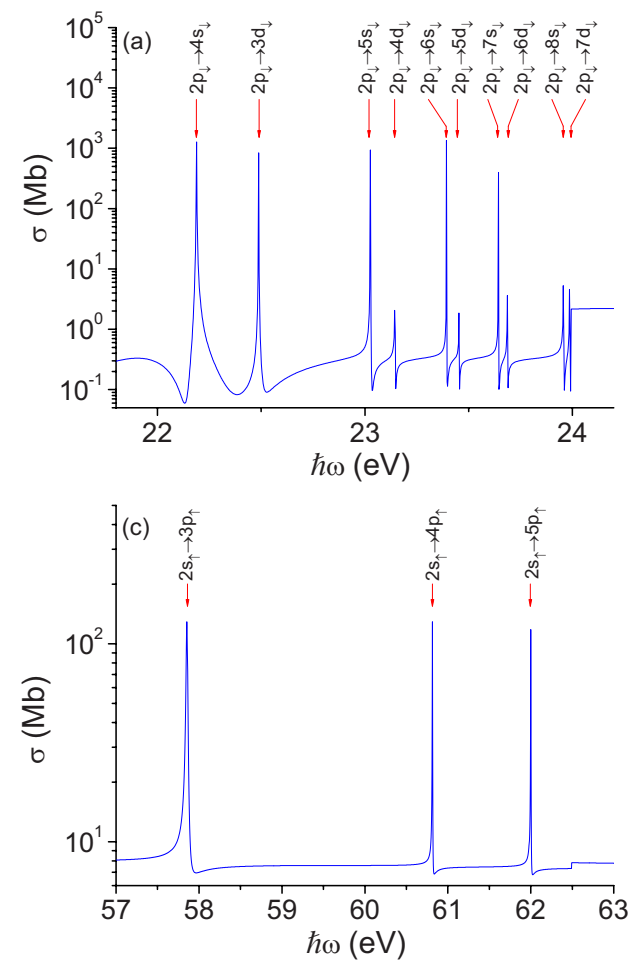

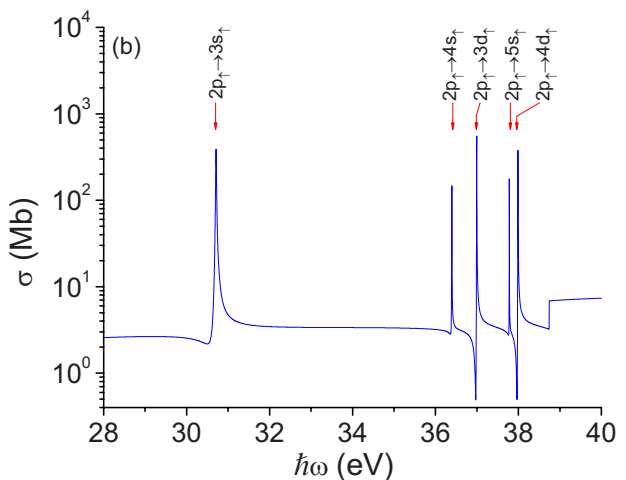

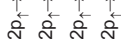

1.

FIG. 7. (Color online) Autoionization resonances in the total photoionization cross sections of $\mathrm{Ne}$ inner-shell excited state $1 s_{\uparrow} 1 s_{\downarrow} 2 s_{\uparrow} 2 p_{\uparrow}^{3} 2 p_{\downarrow}^{3} 3 s_{\downarrow}$ : (a) The resonances produced by the transitions $2 p_{\downarrow} \rightarrow n s_{\downarrow}$ and $n^{\prime} d_{\downarrow}$ with $n \geq 4$ and $n^{\prime} \geq 3$. (b) The resonances produced by the transitions $2 p_{\uparrow}$ $\rightarrow n s_{\uparrow}$ and $n d_{\uparrow}$ with $n \geq 3$. (c) The resonances produced by the transitions $2 s_{\uparrow} \rightarrow n p_{\uparrow}$ with $n \geq 3$. 
TABLE III. Autoionization resonance peak positions (in $\mathrm{eV}$ ) for the photoionizations from Ne inner-shell excited states $\left\{1 s_{\uparrow} 1 s_{\downarrow} 2 s_{\uparrow} 2 p_{\uparrow}^{3} 2 p_{\downarrow}^{3}\right\} n l_{\downarrow}$ with $n=3-4$ and $l=0-2$.

\begin{tabular}{|c|c|c|c|c|c|c|}
\hline \multirow[b]{2}{*}{ Resonant transition } & \multicolumn{6}{|c|}{ Initial states } \\
\hline & \{\}$^{\mathrm{a}} 3 s_{\downarrow}$ & \{\} $3 p_{\downarrow}$ & \{\} $3 d_{\downarrow}$ & \{\} $4 s_{\downarrow}$ & \{\} $4 p_{\downarrow}$ & \{\} $4 d_{\downarrow}$ \\
\hline $2 p_{\downarrow} \rightarrow 3 s_{\downarrow}$ & & 21.655 & 23.821 & 21.478 & 22.681 & 24.128 \\
\hline $4 s_{\downarrow}$ & 22.188 & 24.648 & 26.738 & & 25.356 & 27.086 \\
\hline $5 s_{\downarrow}$ & 23.026 & 25.595 & 27.742 & 24.626 & 26.245 & 27.973 \\
\hline $6 s_{\downarrow}$ & 23.394 & 25.998 & 28.167 & 24.994 & 26.656 & 28.403 \\
\hline $7 s_{\downarrow}$ & 23.644 & 26.215 & 28.381 & 25.187 & 26.866 & 28.629 \\
\hline $8 s_{\downarrow}$ & & 26.411 & 28.509 & 25.328 & 26.988 & 28.756 \\
\hline $9 s_{\downarrow}$ & & & 28.624 & 25.499 & 27.094 & 28.841 \\
\hline $10 s_{\downarrow}$ & & & 28.768 & & 27.224 & 28.947 \\
\hline $3 d_{\downarrow}$ & 22.488 & 24.923 & 27.141 & 24.163 & 25.739 & 27.508 \\
\hline $4 d_{\downarrow}$ & 23.143 & 25.720 & 27.908 & 24.784 & 26.398 & 28.150 \\
\hline $5 d_{\downarrow}$ & 23.453 & 26.060 & 28.248 & 25.073 & 26.730 & 28.493 \\
\hline $6 d_{\downarrow}$ & 23.686 & 26.254 & 28.427 & 25.233 & 26.907 & 28.678 \\
\hline $7 d_{\downarrow}$ & & 26.444 & 28.542 & 25.369 & 27.015 & 28.790 \\
\hline $8 d_{\downarrow}$ & & & 28.654 & 25.543 & 27.118 & 28.866 \\
\hline $9 d_{\downarrow}$ & & & 28.801 & & 27.249 & 29.048 \\
\hline $2 p_{\uparrow} \rightarrow 3 s_{\uparrow}$ & 30.711 & 30.942 & 31.198 & 31.122 & 31.135 & 31.201 \\
\hline $4 s_{\uparrow}$ & 36.393 & 37.647 & 39.089 & 38.904 & 39.002 & 39.263 \\
\hline $5 s_{\uparrow}$ & 37.780 & 39.152 & 41.141 & 41.176 & 41.440 & 41.821 \\
\hline $6 s_{\uparrow}$ & & & & & 42.937 & 43.440 \\
\hline $3 d_{\uparrow}$ & 36.994 & 38.088 & 39.037 & 38.842 & 38.918 & 38.108 \\
\hline $4 d_{\uparrow}$ & 37.987 & 39.288 & 41.206 & 41.127 & 41.421 & 41.750 \\
\hline $5 d_{\uparrow}$ & & & 42.412 & 42.390 & 42.725 & 43.190 \\
\hline $2 s_{\uparrow} \rightarrow 3 p_{\uparrow}$ & 57.854 & 58.344 & 58.768 & 58.638 & 58.676 & 58.785 \\
\hline $4 p_{\uparrow}$ & 60.815 & 62.154 & 63.862 & 63.729 & 63.865 & 64.151 \\
\hline $5 p_{\uparrow}$ & 61.998 & 63.375 & 65.351 & 65.337 & 65.686 & 66.107 \\
\hline
\end{tabular}

$\overline{\bar{a}}\{\}=1 s_{\uparrow} 1 s_{\downarrow} 2 s_{\uparrow} 2 p_{\uparrow}^{3} 2 p_{\downarrow}^{3}$ is the abbreviation of the core electron configuration.

those produced by the transitions from the same electron orbital such as those shown in Figs. 5(a) and 5(b), are no longer the standard Fano profile. Thus it is impossible to accurately evaluate autoionization resonance profile parameters by fitting the resonances to Fano profile. However, we can still estimate the peak position for each autoionization resonance very well. In Table II, we list the peak positions of autoionization resonances for the photoionization from $\mathrm{Ne}$ outer-shell excited states $1 s_{\downarrow} 1 s_{\uparrow} 2 s_{\downarrow} 2 s_{\uparrow} 2 p_{\uparrow}^{3} 2 p_{\uparrow}^{2} n l_{\uparrow}$ with $n$ $=3-4$ and $l=0-2$, where \{\}$=1 s_{\downarrow} 1 s_{\uparrow} 2 s_{\downarrow} 2 s_{\uparrow} 2 p_{\downarrow}^{3} 2 p_{\uparrow}^{2}$ is the abbreviation of the core configuration.

One of the important physical processes during the photoionization is orbital relaxation [44]. Due to the orbital relaxation the peak position of an autoionization resonance is different from the orbital energy difference between the two unperturbed electron orbitals involved. For example, for the excited state $1 s_{\downarrow} 1 s_{\uparrow} 2 s_{\downarrow} 2 s_{\uparrow} 2 p_{\downarrow}^{3} 2 p_{\uparrow}^{2} 3 s_{\uparrow}$, the orbital energy difference between the unperturbed $2 p_{\uparrow}$ and $2 s_{\uparrow}$ electron orbit- als is $23.484 \mathrm{eV}$ while the peak of the autoionization resonance produced by the transition $2 s_{\uparrow} \rightarrow 2 p_{\uparrow}$ is located at $22.994 \mathrm{eV}$. This peak shifts about $0.490 \mathrm{eV}$ toward the lower energy with respect to the unperturbed orbital energy difference. Our calculation shows that for an autoionization resonance produced by the transition from an inner-shell electron orbital to an autoionization electron orbital, the deeper of the inner-shell electron orbital and the lower of the autoionization electron orbital, the larger of the relaxation effect.

\section{Photoionization from inner-shell excited states of $\mathrm{Ne}$}

As another application we extend the proposed approach to the calculation of PICS of Ne inner-shell excited states. In Fig. 6 we plot the total PICS of TDSLHF and TISLHF methods in the solid and dashed lines, respectively, for the photoionization from $\mathrm{Ne}$ inner-shell excited state $1 s_{\uparrow} 1 s_{\downarrow} 2 s_{\uparrow} 2 p_{\uparrow}^{3} 2 p_{\downarrow}^{3} 3 s_{\downarrow}$. The PICS can be divided into four re- 
gions A-D. Each region starts from the IT of an electron orbital and ends at the IT of next lower-energy electron orbital. It is shown that the TISLHF PICS are structureless apart from a stepwise enhancement at each IT. The TDSLHF PICS, however, contain several series sharp autoionization resonances, as shown in Fig. 7.

The region A starts from the IT of $3 s_{\downarrow}$ electron and ends at the IT of $2 p_{\downarrow}$ electron followed by two series of autoionization resonances, as shown in Fig. 7(a). The two series resonances are produced by the transitions of a $2 p$ electron to the higher bound $s_{\downarrow}$ and $d_{\downarrow}$ orbitals and converge to the IT of $2 p_{\downarrow}$ electron. The cross sections near the IT of $3 s_{\downarrow}$ electron again decrease with photon energy. The region B starts from the IT of $2 p_{\downarrow}$ electron and ends at the IT of $2 p_{\uparrow}$ electron. In this region there are also two series of autoionization resonances. They are produced by the transitions of a $2 p_{\uparrow}$ electron to the higher bound $s_{\uparrow}$ and $d_{\uparrow}$ orbitals and converge to the IT of $2 p_{\uparrow}$ electron, as shown in Fig. 7(b). The region $\mathrm{C}$ begins with the IT of $2 p_{\uparrow}$ electron and ends at the IT of $2 s_{\uparrow}$ electron. In this region, there is a series of autoionization resonances induced by the transitions of the $2 s_{\uparrow}$ electron to the higher bound $p_{\uparrow}$ orbitals, as shown in Fig. 7(c). The last region D starts from the IT of $2 s_{\uparrow}$ electron and ends at the maximum energy shown in Fig. 6. In this region, the PICS are structureless since the autoionization resonances will occur at very high energy.

We have also estimated the peak positions for the autoionization resonances. In Table III, we list the peak positions of autoionization resonances for the photoionization from $\mathrm{Ne}$ inner-shell excited states $1 s_{\uparrow} 1 s_{\downarrow} 2 s_{\uparrow} 2 p_{\uparrow}^{3} 2 p_{\downarrow}^{3} n l_{\downarrow}$ for $n=3-4$ and $l=0-2$. Our calculation shows again that for an autoionization resonance due to the transition from an inner-shell electron orbital to an autoionization electron orbital, the deeper of the inner-shell electron orbital and the lower of the autoionization electron orbital, the larger of the relaxation effect.

\section{CONCLUSION}

In this paper, we propose a time-dependent localized Hartree-Fock density-functional linear response approach for the treatment of photoionization of atomic systems. In this approach, the occupied electron orbitals are calculated by solving the KS equation with SLHF exchange potential, the complex susceptibility is calculated by using the occupied electron orbitals and corresponding Green's functions, and the PICS are calculated by using the susceptibility. The relaxation of electron orbitals is taken into account through the Green's functions. To remove the possible reflection of the wave function on the boundary an absorber is imposed for each outgoing wave function in the calculation. The absorber is characterized by an absorptive potential with a linear dependence of coordinates. Since the SLHF exchange potential has a correct long-range behavior and can be used to accurately calculate the electron orbitals of atomic excited states, the proposed approach is suitable for the calculation of photoionization from excited states of atomic systems. We have applied this approach to the calculation of PICS of $\mathrm{Ne}$ ground state. The results are in agreement with available experimental and have comparable accuracies with other $a b$ initio theoretical results. We have also extended this approach to the computation of PICS of Ne excited states. The total PICS can be divided into several regions. Each region starts from the IT of an electron orbital and ends at the IT of the next lower-energy electron orbital. In each region there is one or two series of autoionization resonances produced by the resonant transitions of an electron from inner-shell electron orbitals to autoionization electron orbitals with higher energies. For the autoionization resonances produced by the transitions from inner-shell electron orbitals to autoionization electron orbitals, the deeper of the inner-shell electron orbitals and the lower of the autoionization electron orbitals, the larger of the orbital relaxation effect.

\section{ACKNOWLEDGMENTS}

This work was partially supported by the Chemical Sciences, Geosciences, and Biosciences Division of the Office of Basic Energy Sciences, Office of Science, U.S. Department of Energy and by the National Science Foundation.
[1] P. Hohenberg and W. Kohn, Phys. Rev. 136, B864 (1964).

[2] W. Kohn and L. J. Sham, Phys. Rev. 140, A1133 (1965).

[3] A. Zangwill and P. Soven, Phys. Rev. A 21, 1561 (1980).

[4] Z. H. Levine and P. Soven, Phys. Rev. A 29, 625 (1984).

[5] Z. H. Levine, Phys. Rev. A 36, 1181 (1987).

[6] X. M. Tong and Shih-I Chu, Phys. Rev. A 55, 3406 (1997).

[7] M. Stener, P. Decleva, and A. Lisini, J. Phys. B 28, 4973 (1995).

[8] A. S. Choe, B. Yoo, and J. Lee, J. Phys. B 26, 4099 (1993).

[9] M. Stener and P. Decleva, J. Chem. Phys. 112, 10871 (2000).

[10] M. Stener, P. Decleva, and A. Görling, J. Chem. Phys. 114, 7816 (2001).

[11] M. J. Stott and E. Zaremba, Phys. Rev. A 21, 12 (1980).

[12] G. D. Mahan, Phys. Rev. A 22, 1780 (1980).

[13] S. J. A. van Gisbergen, J. G. Snijders, and E. J. Baerends, J.
Chem. Phys. 103, 9347 (1995).

[14] S. J. A. van Gisbergen, V. P. Osinga, O. V. Gritsenko, R. van Leeuwen, J. G. Snijders, and E. J. Baerends, J. Chem. Phys. 105, 3142 (1996).

[15] C. Jamorski, M. E. Casida, and D. R. Salahub, J. Chem. Phys. 104, 5134 (1996).

[16] R. M. Dreizler and E. K. U. Gross, Density Functional Theory: An Approach to the Quantum Many-Body Problem (SpringerVerlag, Berlin, 1990).

[17] R. G. Parr and W. Yang, Density-Functional Theory of Atoms and Molecules (Oxford University Press, New York, 1989).

[18] A. D. Becke, Phys. Rev. A 38, 3098 (1988).

[19] J. P. Perdew and Y. Wang, Phys. Rev. B 33, 8800 (1986).

[20] F. Della Sala and A. Görling, J. Chem. Phys. 115, 5718 (2001). 
[21] Z. Zhou and Shih-I Chu, Phys. Rev. A 71, 022513 (2005).

[22] J. C. Slater, Quantum Theory of Atomic Structure (McGrawHill, New York, 1960), Vol. II.

[23] Z. Zhou and Shih-I Chu, Phys. Rev. A 75, 014501 (2007).

[24] Z. Zhou and S. I. Chu, J. Phys. B 40, 4379 (2007).

[25] R. Kubo, J. Phys. Soc. Jpn. 12, 570 (1957).

[26] F. Della Sala and A. Görling, Phys. Rev. Lett. 89, 033003 (2002).

[27] C. Lee, W. Yang, and R. G. Parr, Phys. Rev. B 37, 785 (1988).

[28] J. Wang, Shih-I Chu, and C. Laughlin, Phys. Rev. A 50, 3208 (1994).

[29] X. Chu and Shih-I Chu, Phys. Rev. A 63, 023411 (2001).

[30] S.-I. Chu, J. Chem. Phys. 123, 062207 (2005).

[31] D. Neuhasuer and M. Baer, J. Chem. Phys. 90, 4351 (1989).

[32] M. S. Child, Mol. Phys. 72, 89 (1991).

[33] T. Nakatsukasa and K. Yabana, J. Chem. Phys. 114, 2550 (2001).

[34] W. F. Chan, G. Cooper, X. Guo, and C. E. Brion, Phys. Rev. A 45, 1420 (1992).
[35] J. A. R. Samson and W. C. Stolte, J. Electron Spectrosc. Relat. Phenom. 123, 265 (2002).

[36] U. Fano and J. W. Cooper, Phys. Rev. 137, A1364 (1965).

[37] K. Codling, R. P. Madden, and D. L. Ederer, Phys. Rev. 155, 26 (1967)

[38] K. Schulz, M. Domke, R. Puttner, A. Gutierrez, G. Kaindl, G. Miecznik, and C. H. Greene, Phys. Rev. A 54, 3095 (1996).

[39] K. D. Sevier, At. Data Nucl. Data Tables 24, 323 (1979).

[40] B. J. Claessens, J. P. Ashmore, R. T. Sang, W. R. MacGillivray, H. C. W. Beijerinck, and E. J. D. Vredenbregt, Phys. Rev. A 73, 012706 (2006).

[41] R. Kau, I. D. Petrovy, V. L. Sukhorukovy, and H. Hotop, J. Phys. B 29, 5673 (1996).

[42] C. Duzy and H. A. Hyman, Phys. Rev. A 22, 1878 (1980).

[43] T. N. Chang and Y. S. Kim, Phys. Rev. A 26, 2728 (1982).

[44] A. U. Hazi and T. N. Rescigno, Phys. Rev. A 16, 2376 (1977).

[45] K. J. McCann and M. R. Flannery, Appl. Phys. Lett. 31, 599 (1977). 